\title{
Synchronization unveils the organization of ecological networks with positive and negative interactions
}

\author{
Andrea Girón, ${ }^{1}$ Hugo Saiz, ${ }^{2,3}$ Flora S. Bacelar, ${ }^{4}$ Roberto F. S. Andrade, ${ }^{4}$ \\ and Jesús Gómez-Gardeñes ${ }^{1,5}$ \\ ${ }^{1}$ Department of Condensed Matter Physics, University of Zaragoza, E-50009 Zaragoza, Spain \\ ${ }^{2}$ UMR CNRS 6553 Ecosystems-Biodiversity-Evolution, University of Rennes 1, Campus de Beaulieu, \\ Bâtiment 14A, 35042 Rennes Cedex, France \\ ${ }^{3}$ Instituto Pirenaico de Ecología, Avenida Montañana 1005, E-50059 Zaragoza, Spain \\ ${ }^{4}$ Instituto de Fisica, Universidade Federal da Bahia, 40210-340 Salvador, Brazil \\ ${ }^{5}$ Institute for Biocomputation and Physics of Complex Systems (BIFI), University of Zaragoza, \\ E-50018 Zaragoza, Spain
}

(Received 2 February 2016; accepted 28 March 2016; published online 2 June 2016)

\begin{abstract}
Network science has helped to understand the organization principles of the interactions among the constituents of large complex systems. However, recently, the high resolution of the data sets collected has allowed to capture the different types of interactions coexisting within the same system. A particularly important example is that of systems with positive and negative interactions, a usual feature appearing in social, neural, and ecological systems. The interplay of links of opposite sign presents natural difficulties for generalizing typical concepts and tools applied to unsigned networks and, moreover, poses some questions intrinsic to the signed nature of the network, such as how are negative interactions balanced by positive ones so to allow the coexistence and survival of competitors/foes within the same system? Here, we show that synchronization phenomenon is an ideal benchmark for uncovering such balance and, as a byproduct, to assess which nodes play a critical role in the overall organization of the system. We illustrate our findings with the analysis of synthetic and real ecological networks in which facilitation and competitive interactions coexist. Published by AIP Publishing.
\end{abstract}

[http://dx.doi.org/10.1063/1.4952960]

In the last decade, network science has provided the ideal benchmark to encode, analyze, and understand the complex relationships that are established in large scale systems of nature as disparate as the Internet or the brain. As databases become more abundant and complete, we face the challenge of analyzing networks containing multiple types of connections. Here, we tackle the case of signed networks, where the interactions can be either positive or negative. On one hand, we illustrate how synchronization processes capture the organization of this kind of graph into a set of modules interacting negatively among them. With this in mind, we apply the synchronization benchmark to real systems. In particular, we characterize the ecological balance between facilitation and competition in plant communities and analyze the role of species in their organization.

\section{INTRODUCTION}

Synchronization is perhaps the most paradigmatic example of collective behavior as it is recurrently found at different levels of complexity. ${ }^{1-3}$ In fact, the emergence of spontaneous synchronization in systems of coupled dynamical units is at the core of many coordinated tasks, from cognitive processes in the brain to the unfolding of collective behaviors in social systems. ${ }^{4-6}$ In the last decade, network theory has unveiled that the topology of the interactions in a complex system has important effects on the development of collective behaviors. ${ }^{5,7}$ Following this direction, the study of synchronization in networks has attracted a lot of attention ${ }^{8}$ to shed light on the role that the network structure plays on the emergence of synchronized states. ${ }^{9-17}$

A typical setting of the former works consists of associating a dynamical system to each node, whereas the couplings between pairs of dynamical units are mediated by the links of the network. However, other studies have also covered adaptive networks ${ }^{18}$ whose structure is shaped by the microscopic synchronization patterns ${ }^{19-22}$ or systems of mobile oscillators moving in a continuous space. ${ }^{23-27}$ With relatively few exceptions, ${ }^{28-36}$ the hypothesis of these works is that the interactions between units are positive, so that the existence of a link between two coupled dynamical systems implies that they are prone to synchronize as the interaction between them is increased.

In this work, we address the scenario in which positive and negative interactions between nodes coexist in the same network. In this way, the increase of the interaction strength causes the attraction among those dynamical units interacting via positive links and a repulsive effect between those connected through negative edges. As shown recently by Anderson and co-workers, ${ }^{36}$ the stable equilibrium reached consists of a dynamical partition of the network in which nodes sharing positive connections synchronize together while they avoid being dynamically close to those with whom negative interactions are at work. Here, our goal is to use this partition to extract information about the 
organization of networks for which positive and negative relationships between nodes interplay. In particular, we will analyze plant communities where usually both facilitation (positive interaction) and competition (negative interaction) coexist, ${ }^{37}$ playing a key role in the structure and organization of these communities. ${ }^{38-41}$ We will show that synchronization provides an efficient way to unveil how species sharing facilitative interactions group into blocks, allowing the identification of the species that play a major role in the structure of the plant community.

The article is organized as follows. First, in Secs. II and III, we review the main results about the Kuramoto model (KM) of coupled phase oscillators and its studies in signed networks, respectively. Then, in Sec. IV, we analyze a toy model in which two networked systems of attractively coupled oscillators interact repulsively. This allows us to tackle the characterization of real plant communities in Sec. V, exploring the emergence of facilitation groups and the competitive interactions established among them. Finally, in Sec. VI, we round off the article by drawing the concluding remarks.

\section{KURAMOTO MODEL}

In the following, we rely on the paradigmatic Kuramoto model $^{42-44}(\mathrm{KM})$ of coupled limit circle oscillators, as it is the most used dynamical framework for the study of synchronization phenomena in complex networks. ${ }^{8}$ In a complex network of size $N$, the Kuramoto model considers that each node $i$ is characterized by a phase $\theta_{i} \in(0,2 \pi]$ whose time evolution is given by

$$
\dot{\theta}_{i}=\omega_{i}+\lambda \sum_{j=1}^{N} A_{i j} \sin \left(\theta_{j}-\theta_{i}\right) .
$$

The above equation thus describes a set of phase-oscillators coupled with uniform strength $\lambda$ as dictated by the adjacency matrix of the network $\mathbf{A}$, whose terms are $A_{i j}=A_{j i}=1$ if nodes $i$ and $j$ are connected and $A_{i j}=0$ otherwise. Note that, in the original Kuramoto model, ${ }^{42}$ each node $i$ interacts with all the others nodes, so that $A_{i j}=\left(1-\delta_{i j}\right)$. The natural frequencies of the oscillators, $\left\{\omega_{i}\right\}$, are, in principle, different and they are assigned following a frequency distribution $g(\omega)$ that is usually considered to be uni-modal and even around the mean frequency $\Omega$ of the population.

In the uncoupled regime $(\lambda=0)$, each node $i$ describes limit-cycle oscillations with characteristic frequency $\omega_{i}$. However, by increasing the coupling strength $\lambda$, the network undergoes a phase transition at some critical value $\lambda_{c}$ that, for an arbitrary network topology described by an adjacency matrix $\mathbf{A}$, is given by $\lambda_{c}=2 /[\pi g(\Omega) \Lambda(\mathbf{A})],{ }^{45}$ where $g(\Omega)$ is the density of oscillators with natural frequency equal to the average one, $\Omega$, and $\Lambda(\mathbf{A})$ is the maximum eigenvalue of the adjacency matrix.

To monitor the synchronization transition, Kuramoto introduced the following order parameter

$$
r(t) \mathrm{e}^{\mathrm{i} \Psi(t)}=\frac{1}{N} \sum_{j=1}^{N} \mathrm{e}^{\mathrm{i} \theta_{j}(t)},
$$

which is the averaged sum of the unit vectors associated with the phases of each oscillator in the complex plane. The modulus of the resulting complex number is the order parameter, $r(t) \in[0,1]$, that measures the coherence of the collective motion of the oscillators. In this way, $r=1$ when the network is fully synchronized whereas $r=0$ stands for the incoherent solution. In addition, the value of $\Psi(t)$ accounts for the average phase of the ensemble of oscillators.

\section{KURAMOTO MODEL IN SIGNED NETWORKS}

The extension of the KM, Eq. (1), to address the possibility of positive and negative interactions was initially proposed by Daido ${ }^{28}$ more than twenty years ago. There he considered an all-to-all Kuramoto model with random interaction weights (or equivalently random entries $A_{i j}$ ) including positive and negative ones. From this study, a debate about the glassy behavior of the system was opened and its solution still remains unclear. ${ }^{29,30}$ Recently, the combination of positive and negative interactions in a network of Kuramoto oscillators has recovered the attention of researchers. ${ }^{32-35}$ For instance, in Ref. 32, the authors show that the addition of negative links to networks can enhance the stability of the synchronized state, while Hong and Strogatz ${ }^{33-35}$ studied two different coarse-grained versions of the Daido model to shed light on the aforementioned debate.

Here, we will revisit the KM in the following way. Instead of working with weighted adjacency matrices, we allow the weights of the links to take one out of three values. Namely, $A_{i j}=A_{j i}=1$ if the interaction between $i$ and $j$ is positive, $A_{i j}=A_{j i}=-1$ if the link between $i$ and $j$ is negative, and $A_{i j}=A_{j i}=0$ if no interaction between $i$ and $j$ is present. This is equivalent to work with two adjacency matrices, one encoding all the positive interactions, $\mathbf{A}^{+}$, and another one, $\mathbf{A}^{-}$, accounting for the negative ones. In this way, we have $\mathbf{A}=\mathbf{A}^{+}-\mathbf{A}^{-}$, and the KM, Eq. (1), can be rewritten as:

$$
\dot{\theta}_{i}=\omega_{i}+\lambda \sum_{j=1}^{N}\left[A_{i j}^{+} \sin \left(\theta_{j}-\theta_{i}\right)+A_{i j}^{-} \sin \left(\theta_{i}-\theta_{j}\right)\right] .
$$

The above equation is the most simple generalization of the KM in a complex network to the case of signed graphs. However, it can be applied to many real situations in which positive and negative interactions coexist within the same system. A familiar case is that of social networks, in which the acquaintances of an individual can be classified as friends and foes. In this latter case, the focus has been the test of social balance theory ${ }^{46,47}$ in large scale social networks ${ }^{48}$ where complex structures mixing positive and negative ties appear.

As anticipated, here, we will focus on a different real world example where the interplay between negative and positive interactions appears naturally: ecological networks. We rely on recent data sets about the interaction between vegetal specie ${ }^{49,50}$ in which the nature of the interactions between the different species was derived by analyzing their local spatial association patterns. Although an approximation to real biotic interactions, spatial association between plant 
species is considered as a valid proxy for studies including all species in a community. ${ }^{39,51,52}$ These interactions can be of three types: neutral (no interaction, species associate at random in space), facilitation (positive interaction, species co-occur in space more than expected), or competition (negative interaction, species segregate in space more than expected). In this way, the two matrices $\mathbf{A}^{+}$and $\mathbf{A}^{-}$can be constructed straightforward.

\section{SIMPLE SYSTEM: TWO INTERCONNECTED NETWORKS}

The KM has been used to unveil the modular organization of unsigned networks ${ }^{12,53-56}$ relying on the observation that synchronization is first attained among those nodes belonging to the same module, or community, and then spreads to the rest of the network. This provides a fast and an efficient way to detect the modular structure of the network. For signed networks, Anderson et al. ${ }^{36}$ analyzed the behavior of a system composed of $M$ large groups of coupled Kumamoto oscillators, so that the interactions within members of the same group are positive whereas those between oscillators belonging to different groups are negative. To illustrate their results, we consider here the simplest case of $M=2$ (analyzed in Refs. 57-59 for unsigned networks) groups, see Fig. 1. Thus, as in Ref. 36, we assume that there is a perfect partition of the system into two sub-populations that we know a priori. Our aim is to observe how synchronization patterns are organized so as to gain insight for the application to real systems in which the sub-population structure, if any, is unknown.

As shown in Fig. 1, we consider a toy multilayer network $^{60,61}$ composed of two fully connected graphs of identical size $N$, while the probability that two nodes belonging to different networks are connected is equal to $p$. In this way, each network contains $N(N-1) / 2$ positive links while, on average, the two networks are interconnected via $p N^{2}$ links. Thus, the system contains $N^{2}$ positive and $p N^{2}$ negative links. Considering the probability of connection $p$ between two nodes of different populations, the KM for the system of two interconnected networks can be approximated as:

$$
\dot{\theta}_{i}^{\alpha}=\omega_{i}^{\alpha}+\lambda \sum_{j=1}^{N} \sin \left(\theta_{j}^{\alpha}-\theta_{i}^{\alpha}\right)-p \lambda \sum_{j=1}^{N} \sin \left(\theta_{j}^{\beta}-\theta_{i}^{\alpha}\right),
$$

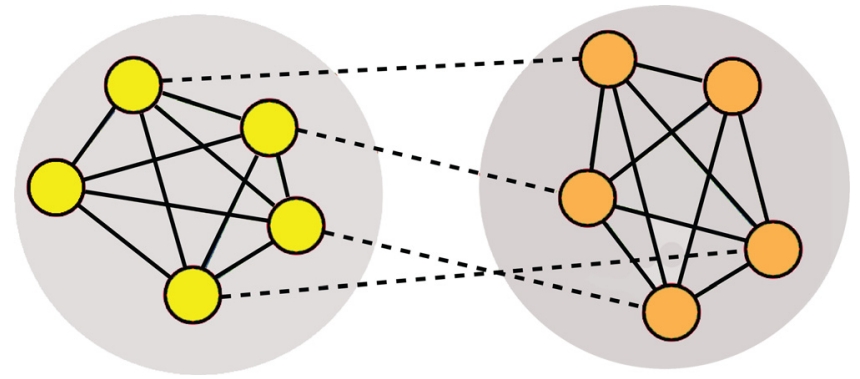

FIG. 1. Two interconnected networks of 5 nodes each. Each network is a fully connected graph. The links among nodes of the same networks are positive (solid), whereas the 4 links connecting nodes of different networks are negative (dashed). where $\theta_{i}^{\alpha}$ and $\omega_{i}^{\alpha}$ are the phase of oscillator $i(i=1, \ldots, N)$ in population $\alpha$ and its natural frequency, respectively. Note that in Eq. (4) $\alpha=1,2$ and $\beta=2,1$, respectively.

In addition to the Kuramoto order parameter $r$, it is useful to define a synchronization parameter associated with each population $\alpha$. In analogy to Eq. (2), this order parameter reads

$$
r^{\alpha}(t) \mathrm{e}^{\mathrm{i} \Psi^{\alpha}(t)}=\frac{1}{N} \sum_{j=1}^{N} \mathrm{e}^{\mathrm{i} \theta_{j}^{\alpha}(t)}
$$

where $r^{\alpha} \in[0,1]$ accounts for the degree of synchronization in population $\alpha$, whereas $\Psi^{\alpha}$ defines the average phase of oscillators belonging to network $\alpha$. With the help of Eq. (5), we can re-write Eq. (4) as

$$
\dot{\theta}_{i}^{\alpha}=\omega_{i}^{\alpha}+K r^{\alpha} \sin \left(\Psi^{\alpha}-\theta_{i}^{\alpha}\right)-p K r^{\beta} \sin \left(\Psi^{\beta}-\theta_{i}^{\alpha}\right),
$$

where $K=N \lambda$, recovering the usual mean-field form of the $\mathrm{KM}^{42-44}$ Assuming, without loss of generality, a reference frame rotating with the mean frequency $\Omega$ of the system (or analogously considering $\Omega=0$ ) and whose position is set so that the mean phase of the oscillators in one of the networks is $\Psi^{\alpha}=0$, the equation for the locked, $\dot{\theta}_{i}^{\alpha}=0$, oscillators reads

$$
\omega_{i}^{\alpha}=K r^{\alpha} \sin \left(\theta_{i}^{\alpha}\right)+p K r^{\beta} \sin \left(\Psi^{\beta}-\theta_{i}^{\alpha}\right) .
$$

The above equation is only fulfilled for a set of oscillators in each of the populations having a natural frequency close enough to the average one $\Omega=0$.

Due to the high symmetry of the two populations and under the assumption that the frequency distribution is the same for both networks, $g^{\alpha}(\omega)=g^{\beta}(\omega)$, we can establish a map between a locked oscillator $i$ in population $\alpha$, with its counterpart, say $i$, in population $\beta$ so that $\omega_{i}^{\alpha}=\omega_{i}^{\beta}$. In this way, for each pair of locked oscillators, we have

$$
\sin \left(\theta_{i}^{\alpha}\right)+p \sin \left(\theta_{i}^{\beta}\right)=\sin \left(\theta_{i}^{\beta}-\Psi^{\beta}\right)+p \sin \left(\theta_{i}^{\alpha}-\Psi^{\beta}\right),
$$

where we have considered, again invoking the symmetry of the system and that $g^{\alpha}(\omega)=g^{\beta}(\omega)$, that the degree of synchronization of the two populations is identical, $r^{\alpha}=r^{\beta}$, for a given value of $K$. The self-consistent set of equations (8) has two solutions: $\Psi^{\beta}=0\left(\theta_{i}^{\alpha}=\theta_{i}^{\beta}\right)$ and $\Psi^{\beta}=\pi\left(\theta_{i}^{\alpha}=\theta_{i}^{\beta}-\pi\right)$. It can be easily shown (see the Appendix for a simple argument and the supplementary material ${ }^{62}$ for a proof) that the first solution $\left(\Psi^{\alpha}=\Psi^{\beta}=0\right)$ is unstable whereas the second one $\left(\Psi^{\alpha}=0, \Psi^{\beta}=\pi\right)$ is stable, pointing out that although synchronization can be attained within each of the populations they tend to be in anti-phase so that the degree of synchronization of the whole system $r$ falls to zero.

In Fig. 2, we show this phenomenon by considering two networks of $N=500$ nodes and an interconnection probability of $p=0.4$. The natural frequencies are randomly assigned from an homogeneous (flat) distribution within the range $\omega_{i}^{\alpha} \in[-1 / 2,1 / 2]$. In the top panels, (a)-(c), we show the distribution of the phases in the unit circle of the complex plane for $K=0, K=0.5$, and $K=1$. It is shown how 

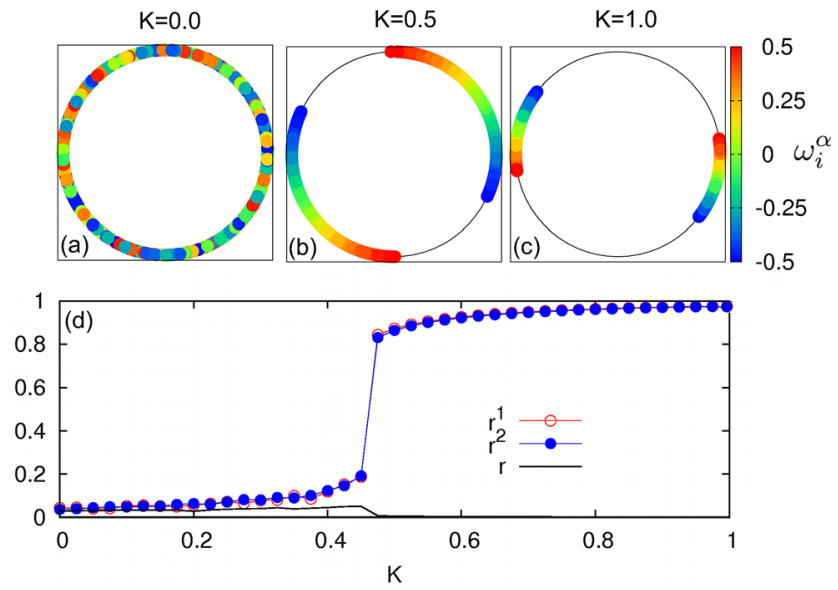

FIG. 2. Panels (a)-(c) show the distribution of the values $e^{\mathrm{i} \theta_{i}^{\alpha}}$ in the unit circle of the $2 N(N=500)$ oscillators for $K=0$ (a), $K=0.5$ (b), and $K=1$ (c). The color of each circle denotes the natural frequency $\omega_{i}^{\alpha}$ of each oscillator: the darker the more different from the average frequency $(\Omega=0)$. In panel (d), we show the evolution of the synchronization of the system, $r$, and each of the networks, $r^{\alpha}(\alpha=1,2)$, as a function of the coupling $K$. The system is composed of two fully connected networks interconnected with probability $p=0.4$.

synchronization within the groups, pointed by the concentration of the circles, is attained as $K$ is increased. From these panels, it is also observed that the synchrony groups are in anti-phase. Panel (d), in the bottom, shows the evolution of the degree of synchronization within the groups, $r^{1}$ and $r^{2}$, and that of the whole system, $r$, as a function of $K$. It becomes clear that the anti-phase alignment of the synchrony groups observed above makes $r$ decrease to 0 while the values $r^{\alpha}$ increase for $K>K_{c} \simeq 0.45$.

Now, we focus on the onset of synchronization $K_{c}$ for the two populations. By considering the stable solution of Eq. (8) in Eq. (6), we can express the evolution for the phase of an oscillator $i$ in population $\alpha$ as:

$$
\dot{\theta}_{i}^{\alpha}=\omega_{i}^{\alpha}-(1+p) K r^{\alpha} \sin \left(\theta_{i}^{\alpha}\right) .
$$

The above equation is exactly the same to the original KM with the exception of the $(1+p)$ correction in the coupling strength. Thus, within a population $\alpha$, synchronization shows up at some critical coupling $\lambda_{c}=K_{c} / N$, where

$$
K_{c}=\frac{K_{c}^{K M}}{(1+p)}=\frac{2}{\pi g(0)(1+p)} .
$$

In Fig. 3, we show the degree of synchronization within a network, $r^{\alpha}$, as a function of the coupling constant $K$ normalized to the corresponding value in the original $\mathrm{KM}, K_{c}^{K M}$. Obviously, for $p=0$, we have two independent fully connected networks, i.e., two systems obeying the original KM, and thus, the onset is reached exactly at $K_{c}^{K M}$. As predicted from Eq. (10) as $p$ increases, so that negative links between networks appear, the onset of synchrony is anticipated, reaching half of the original value, $K_{c}^{K M}$, when $p=1$.

Apart from the knowledge gained from the analysis of the toy multilayer network, the anticipation of the onset of synchronization within each population as $p$ increases constitutes an apparently counterintuitive result: the more

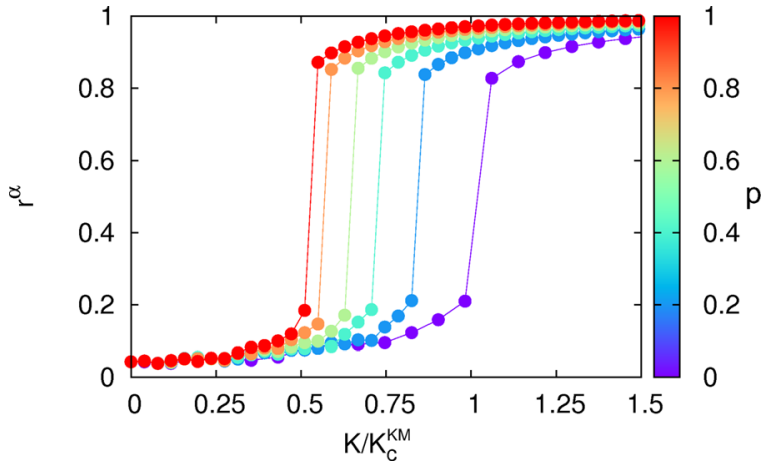

FIG. 3. Degree of synchronization within a population $\alpha$ as a function of $K$ for different values of interconnection probability $p$. The value of $K$ is normalized to that of the original Kuramoto model $K_{c}^{K M}$. The agreement with the theoretical prediction for the synchronization onset, Eq. (10), is excellent. The interconnected system is the same as in Fig. 2.

repulsive links we add the easier synchronization is attained between nodes sharing attractive interactions. Translated into a social context, this implies that two friends tend to become more similar as the number of common foes increases. This result constitutes a nice theoretical example of the importance that negative interactions have on the collective behavior, here synchronization, of complex systems.

\section{UNCOVERING THE STRUCTURE OF REAL SIGNED NETWORKS}

Now, we address the characterization of two real ecological networks in which negative and positive interactions interplay. These networks capture the relationships established between vegetal species by measuring the spatial associations among them. ${ }^{49,50}$ Spatial associations are derived by analyzing the deviations with respect to the expected cooccurrence frequency when plants are randomly distributed in space. In this way, those pairs of species co-occurring more than expected are considered to interact positively (facilitation), whereas those pairs co-occurring less than expected are considered to interact negatively (competition).

The two studied networks correspond to two typical Iberian semi-arid locations, Cabo de Gata (Almería) and Monegros (Aragón), being both placed in Spain. The main properties of both networks are collected in Table I. In both networks, positive interactions dominate over negative ones, a typical property of plant communities under stressful environmental conditions where positive interactions are hypothesized to be more important than negative ones. ${ }^{63,64}$ The main difference between these two networks lies on their different density of links, being the Monegros networks

TABLE I. Main properties of the networks analyzed. For each network (named as the area where data were collected), we show the number of nodes (species), $N$, the average degree of nodes, $\langle k\rangle$, the average number of positive (negative) connections per node, $\langle k\rangle^{+}\left(\langle k\rangle^{-}\right)$, the number of connected components, $N_{c}$, and the size of the largest one, $S$.

\begin{tabular}{lcccccc}
\hline \hline Area & $N$ & $\langle k\rangle$ & $\langle k\rangle^{+}$ & $\langle k\rangle^{-}$ & $N_{c}$ & $S$ \\
\hline Cabo de Gata (Almería) & 42 & 2.476 & 1.381 & 1.095 & 2 & 40 \\
Monegros (Aragón) & 46 & 3.347 & 2.695 & 0.652 & 2 & 44 \\
\hline
\end{tabular}


the most wired one. A possible explanation is the presence of high livestock grazing intensity in the plant community of Cabo de Gata (0.65 ind/ha), for an area where grazing carrying capacity is $0.39-0.57$ ind/ha (Ref. 65). Overgrazing is known to have a deep impact on the structure of semi-arid plant communities, particularly breaking the spatial organization of vegetation patches and randomizing the associations between plant species. ${ }^{66,67}$ Thus, the network is expected to present less linkage density. Nevertheless, the differences between the two networks allow us to validate the partitioning method in real communities with different properties.

To analyze how competitive and facilitation relationships among species interplay and shape the interaction network, we proceed as follows. We set all the initial phases of the oscillators as $\theta_{i}(0)=0 \forall i$ and assign nearly identical natural frequencies $\omega_{i}=0+\xi$ (with $\xi$ being a random variable homogeneously distributed in the interval $\left[-10^{-3}, 10^{-3}\right]$ ). Then, we start to increase adiabatically the value of the interaction coupling $K$ by small increments $\delta K$ and monitor how the dynamical equilibria reached for each value of $K$ evolve from small to large values of the coupling strength. Note that, at variance with the system analyzed in Section IV, here we do not know the partition, if any, of the network so that our goal is to detect how groups show up from the synchronization clusters.

\section{A. Cabo de Gata ecosystem}

In Fig. 4, we analyze the synchronization patterns of the first network (Cabo de Gata). The first insight is provided by the evolution of the degree of synchronization, $r$, of the network as a function of the coupling $K$ (see Fig. 4(a)). Given the initial conditions described above for $K \ll 1$, the oscillators remain fully synchronized $(r=1)$ as a product of the very small deviation in their natural frequencies. However, as $K$ increases, there is a sudden drop of $r$ due to the repulsive effect of negative interactions, and finally, for $K>0.2$, the equilibrium state remains unaltered displaying a degree of synchrony of $r \simeq 0.89$.

Once each dynamical equilibrium is reached for a value of $K$, we look at the distribution of the steady phases $\left\{\theta_{i}^{\star}\right\}$ of the oscillators in the unit circle. In particular, we measure the relative phase of an oscillator $i$ with respect to that of oscillator 1 (the most connected node in the network): $\Delta \theta_{i}=\theta_{i}^{\star}-\theta_{1}^{\star}$. In Fig. 4(b), we observe that as soon as the coupling strength $K$ is large enough, a stable organization of the network into clusters of oscillators having the same dynamical state shows up. According to Fig. 4(b), we can distinguish 6 clusters, denoted from I to VI in the right vertical axis (Fig. 4(b)). At first sight, it becomes clear that all the species in groups II to $\mathrm{V}$ are strongly repelled by cluster I since these latter clusters are placed around $\Delta \theta_{i}=-\pi$. In fact, by looking at the composition of cluster I, we notice that this is composed by only node 1 (the hub of the system) whose interactions are all repulsive. Thus, node 1 appears as a very competitive species which play a central role structuring the plant community.

Taking advantage of the partition of the network provided by the Kuramoto dynamics, we can organize the nodes of the network as in Fig. 4(c) so as to understand the composition and role of the remaining clusters II to VI. First, we notice that all the members of cluster $\mathrm{V}$ are either direct competitors of node 1 (cluster I) or neighbors of these competitors, suggesting a large group of species that associate among them in the spatial gaps left by species 1 . This result agrees well with the distribution shown in Fig. 4(b) since cluster $\mathrm{V}$ is placed just at $\Delta \theta_{i}=-\pi$, whereas clusters II, III, and IV, although also containing competitive interactions with node 1 , are displaced in such a way that $\Delta \theta_{i}>-\pi$ for the nodes they contain. The reason for such displacement comes from the competition of cluster II with cluster VI (composed of nodes 2 and 3) which, in its turn, also competes with cluster I (node 1). In fact, the competition between clusters I, II, and VI manifests in their respective disposition observed in Fig. 4(b) where they appear in such a way that nearly maximizes their relative distance. The organization of these clusters reveals the existence of two other competitors (cluster VI) not as strong as node 1 but with a specific negative effect on the species of cluster II. In principle, if these clusters I, II, and VI had no interactions with clusters IV and III, they would appear placed at $\Delta \theta_{i}=0,-2 \pi / 3$, and $-4 \pi / 3$ respectively. However, cluster II has positive interactions with cluster III which, in its turn, interacts negatively with cluster I. Thus, as a result of the attraction between clusters II and III, the positions of the
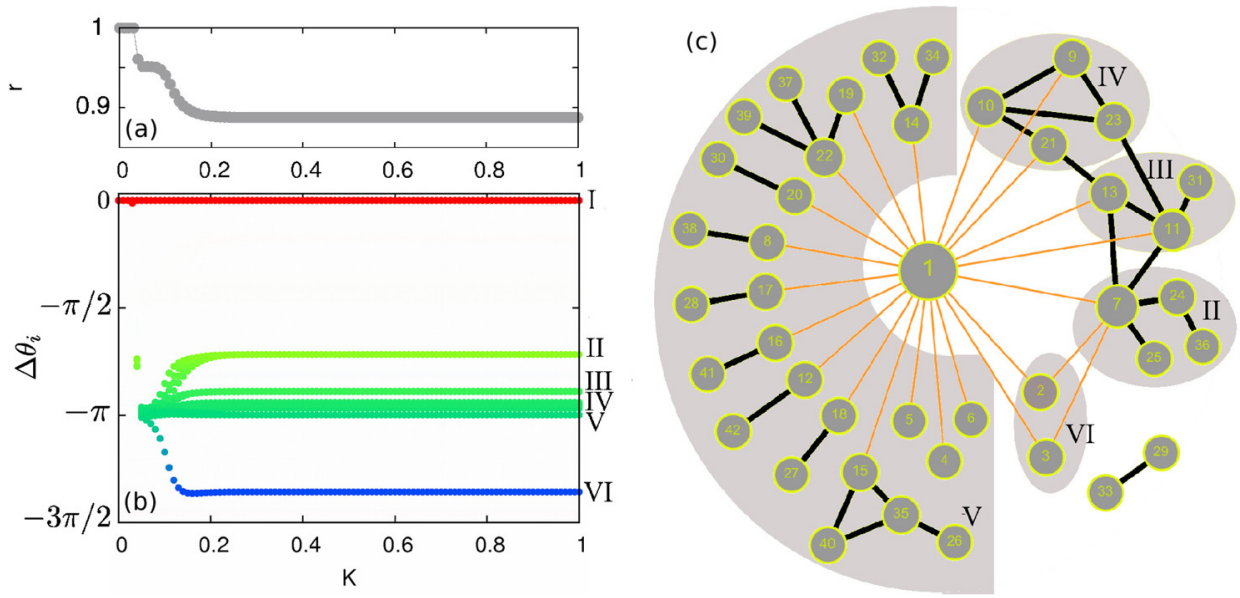

FIG. 4. Analysis of the Cabo de Gata ecosystem. The first panel (a) shows the evolution of the degree of synchronization $r$ of the whole network as a function of the coupling strength $K$. Panel (b) shows the evolution, as a function of $K$, of the phase difference of node $i$ with respect to node $1, \Delta \theta_{i}$. Finally, panel (c) shows the network of interactions [facilitation links in black while negative ones appear in grey (orange in the color version)] by grouping the nodes in six groups as revealed from panel (b). 

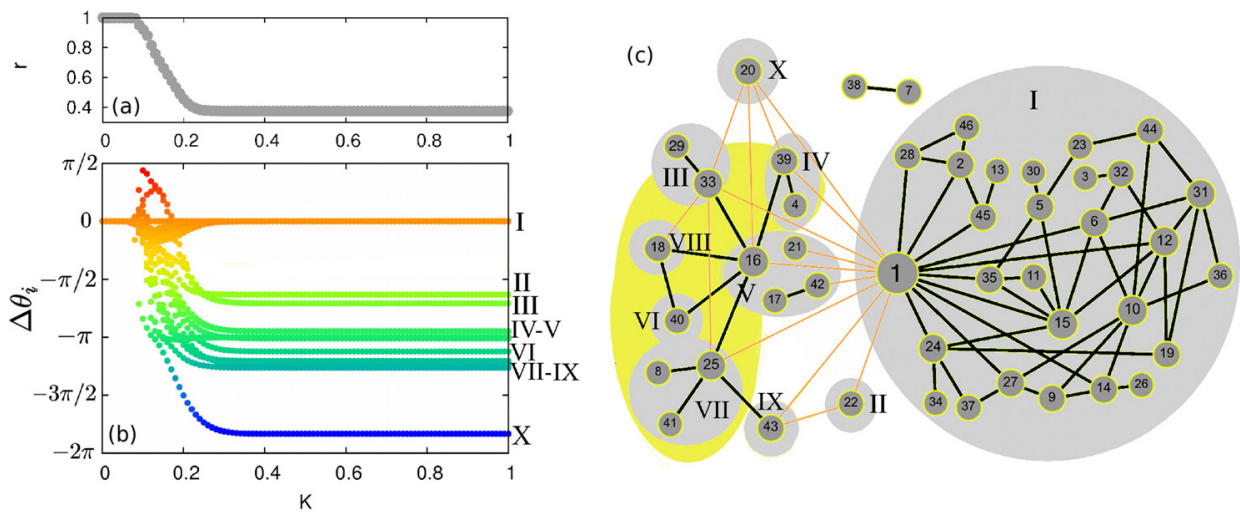

FIG. 5. Analysis of the Monegros ecosystem. As in Fig. 4, the first panel (a) shows the evolution of the degree of synchronization $r$ of the whole network as a function $K$. Panel (b) shows the evolution of $\Delta \theta_{i}$ as a function of $K$, pointing out the existence of 10 groups. Finally, panel (c) shows the network of interactions [facilitation links in black while negative ones appear in grey (orange in the color version)] by grouping the nodes in the 10 groups derived from panel (b). nodes in these clusters appear displaced from $\Delta \theta_{i}=-2 \pi / 3$ and $-\pi$, respectively. Finally, the displacement of nodes in cluster III from $\Delta \theta_{i}=-\pi$ originates a (smaller) displacement of nodes in cluster IV from $\Delta \theta_{i}=-\pi$ due to the facilitation links $(21,13)$ and $(23,11)$.

\section{B. Monegros ecosystem}

Now, we proceed to analyze the second network corresponding to the Monegros location. As shown in Table I and discussed above, this system is richer than that of Cabo de Gata in terms of the number of interactions, and at variance with the first network, facilitation interactions appear significantly more frequently than competitive ones. The analysis is done in the same fashion as for the first network (see Fig. 5). In Fig. 5(a), we show the evolution of the degree of synchronization of the system as a function of $K$. This provides us a first insight about the minimum coupling strength needed to reach an equilibrium in which the competition between negative and positive interactions is manifested. This happens for $K>0.25$. The evolution with $K$ of the phase difference of each node $i$ with respect to the most connected node (again node 1 ), $\Delta \theta_{i}$, is shown in Fig. 5(b) and corroborates that for $K<0.25$ there is a self-organization of the phases into groups that become stable for $K>0.25$.

Fig. 5(b) clearly shows the organization of the species (nodes) into 10 groups (named as I, II,..., X) that are shown in the representation of the network in Fig. 5(c). The first group is composed of node 1 and all the species that interact (directly or indirectly) with it via positive links. Thus, node 1 plays a key role in structuring the community by isolating species in group I from the rest of the system. In this case, node 1 is clearly a nurse species responsible for patch formation. These patches act as shelters against the harsh environmental conditions, increasing the chances of survival of the species in cluster I.

The remaining nodes in the network compete (directly or indirectly) with node 1 and represent the species that are established outside the patches including species 1 . However, this second part of the system appears fragmented into 9 groups due to the internal competition links between the members of these groups. As competitors of node 1, the members of group V appear, as shown in Fig. 5(b), placed at $\Delta \theta_{i}=-\pi$, whereas groups II to $\mathrm{X}$ are displaced from this antagonist position. The reason for the displacement lies on the competitive interactions launched from groups $\mathrm{X}$ and II which are competitors of group I as well. For instance, group $\mathrm{X}$ is competitor of groups I, III, IV, V so that it automatically implies that, considering the position of groups I $\left(\Delta \theta_{i}=0\right)$ and $\mathrm{V}\left(\Delta \theta_{i}=-\pi\right)$, the positions of groups III and IV lie in the range $\Delta \theta_{i} \in(0,-\pi)$ as they are also competitors of group I and are positively linked to group $\mathrm{V}$.

The role of groups III and V would be identical (as given from their respective interactions with groups I, V, and $\mathrm{X})$ if it were not for the competitive interactions that group III shares with groups VII and VIII that, in their turn, are positively connected to group $\mathrm{V}$. These negative links become evident from the position of group III in Fig. 5(b) $\left(\Delta \theta_{i}>-\pi\right)$, whereas group IV, not having these competitive interactions, lies close to group $\mathrm{V}\left(\Delta \theta_{i} \simeq-\pi\right)$. The negative interactions of group III with groups VII and VIII automatically set their position at $\Delta \theta_{i}<-\pi$ while being close to group $\mathrm{V}$ with whom share facilitation links. Moreover, the positive interactions of groups VII and VIII with groups IX and VI, respectively, set the latter close to the former, being group VI the closest to $\Delta \theta_{i}=-\pi$ of the four due to its positive link to group V. On the other hand, group IX is the farthest one due to the negative interaction with group II (another competitor of group I) which, as a consequence, appears in the region $\Delta \theta_{i}>-\pi$.

\section{Unveiling the organization of modules}

With the partition obtained above, one can easily analyze the organization that facilitation and competitive interactions provide to the system. In Fig. 6, we show the coarsegrained representation of the Cabo de Gata (a) and Monegros (b) networks where the nodes account for the detected groups and the interactions between them are positive or negative depending on the nature of the interactions between the nodes each group contains. This picture provides a valuable tool to identify groups of species which present the same interaction behavior in the system. For example, a group of species which share positive interactions represents a particular type of vegetation patch in the community (as group I in the Monegros ecosystem). Let us remark that none of the pairs of connected groups shares mixed interactions, i.e., the links between groups are either positive or negative. Thus, the partitions obtained from synchronization clusters allow us to interpret unambiguously the relationships between the different groups of species in an ecosystem. 

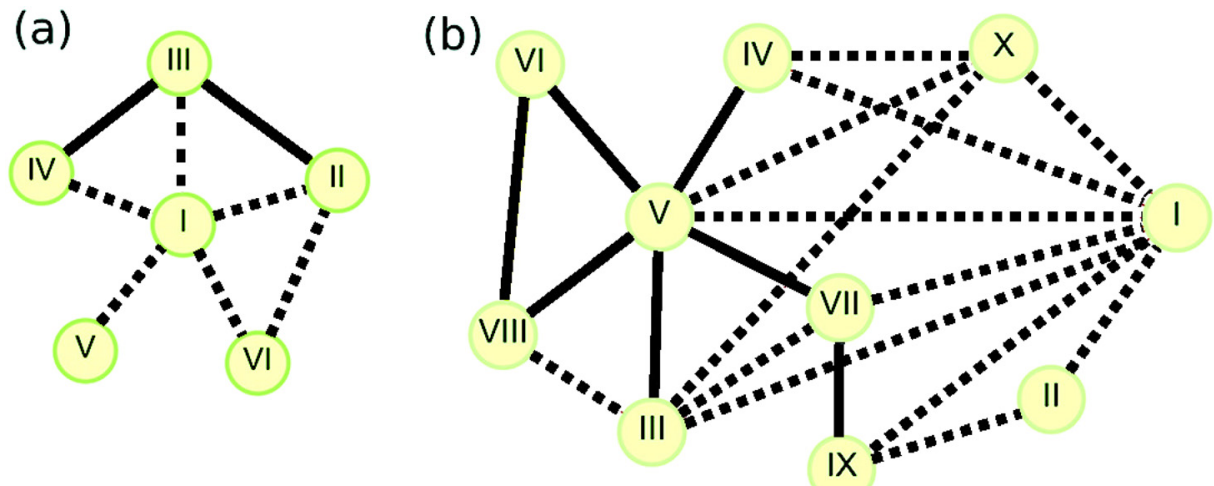

FIG. 6. Coarse-grained description of the Cabo de Gata (a) and Monegros (b) ecosystems as obtained from the Kuramoto partitions. The nodes of these networks represent groups of species whereas a positive (negative) link between a pair of groups exists if, at least, one positive (negative) interaction between two species belonging to each group is present in the original network. Solid (dashed) links represent positive (negative) interactions.

For the first network (see Fig. 6(a)), it is easy to notice the critical role that group VI has on the organization of the network. In fact, by deleting group VI, the role played by II, III, and IV would be identical so that they would merge into one single group, and analogously, this new resulting group would have an analogous role to that of group $\mathrm{V}$ implying a new merging of groups that would turn the system into a network of two competing populations. This conclusion can be easily reached by looking directly into the corresponding network of interactions between species (see Fig. 4(c)). However, the analysis of the Monegros network (see Fig. $5(c)$ ) becomes more difficult.

In ecological terms, the pattern of interactions between the groups in the Monegros ecosystem, shown in Fig. 6(b), suggests the existence of a complex competition pattern among the modules competing with module I, probably related to the particular life histories of the species composing them. Specifically, by considering groups II to X, we observe that groups II and $\mathrm{X}$ are monospecific so that the two species (22 and 20, respectively) establish themselves outside the vegetation patches composed of species belonging to groups I and III to IX. However, from Fig. 6(b), it becomes clear that vegetation patches containing species from groups III to IX can be composed of species belonging to group $\mathrm{V}$ together with some species belonging to groups III, IV, and VI-IX. Specifically, the negative interactions that species in module III share with those in modules VII and VIII suggest that species in these groups limit the establishment of other species when they are already present in the patch.

Interestingly, the coarse-grained network of the Monegros ecosystem (Fig. 6(b)) provides useful insights about the response of the system under certain perturbations. For instance, the aforementioned fragmentation into several groups of the population competing with group I can be seen as a product of the two unbalanced triads, (III-V-VIII) and (III-V-VII), in the coarse grained network. Thus, one can ask what are the effects associated with the removal of group III or, alternatively, the absence of species 33 , as it is the one responsible for the unbalanced triads created in the network of species (Fig. 5(c)). To this aim, we have studied the Kuramoto dynamics with and without species 33 for $K=1$. In this way, we evolve in time the Kuramoto dynamics of the whole system, and at some point, we remove node 33 in order to monitor how a new partition of the network shows up.
In Fig. 7, we report the original equilibrium distribution of the phases of the 44 species in the unit circle in the Monegros system (a) and the one after removing group III (b). The result is that the population passes from being partitioned into 10 groups to have 6 modules. One module (III) is trivially removed, however, as a byproduct other 3 groups have disappeared or, better said, three new groups have emerged from the mixing of six (IV-IX) old ones. The merging of the 6 modules is shown in Fig. 7(c) by monitoring the evolution of $\Delta \theta_{i}$ for the nodes in groups IV-IX. At time $t=0$, we remove group III and subsequently a reorganization of the species into new three groups takes place. The composition of the most populated of the new groups is highlighted in Fig. 5(c), and as shown, it comprises former groups IV, VI, VII, and VIII and species 16 from group V.

In a nutshell, the absence of species 33 creates a much larger coalition of species competing with those contained in group I. This result illustrates the critical role played by group III in the organization of the system and, in particular, in the fragmentation of the original coalition. In ecological terms, the suppression of species 33 would foster the formation of vegetation patches mixing species from former

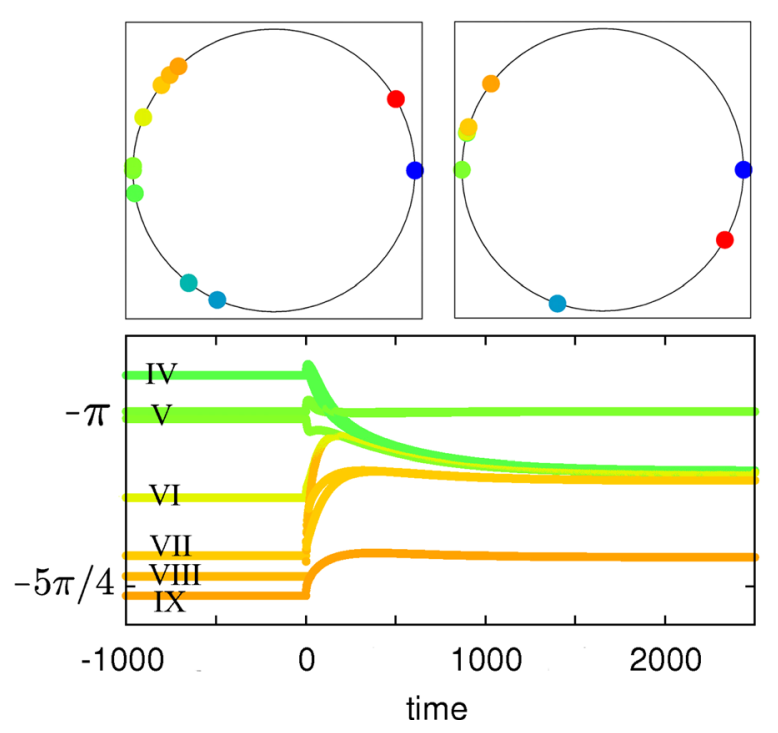

FIG. 7. Panels (a) and (b) show the distribution at equilibrium of the phases of each species in the unit circle. The panels show these distributions in the Monegros ecosystem before (a) and after (b) removing group III. Finally, panel (c) shows the time evolution of the values $\Delta \theta_{i}$ of the nodes in groups IV-IX before $(t<0)$ and after $(t>0)$ removing group III. 
modules IV, VI, VII, and VIII, that, in the original system, were highly conditioned by the presence of species 33 .

\section{CONCLUSIONS}

The analysis carried out in both Cabo de Gata and Monegros locations unveils that the interplay between negative and positive interactions yields an effective partition of signed networks that can be detected by analyzing the synchronization patterns between the nodes. Importantly, this analysis can be done straightforward without any a priori information about the number and size of groups in the system and avoiding computationally expensive optimization algorithms.

The method introduced here is along the same line as the one reported in Refs. 12 and 53-56 for the case of community detection in complex (unsigned) networks by means of the classical attractive Kuramoto model. Very recently, ${ }^{68}$ another dynamical clustering based on the dynamics of a deformed KM has been implemented to analyze the structure of signed social interactions. Here, after illustrating in Sec. IV the patterns that the interplay between attractive and repulsive interactions introduces in the stable equilibrium of Kuramoto oscillators in ideal modular signed networks ${ }^{36}$ by analyzing the case of two interconnected groups; in Secs. $\mathrm{V} \mathrm{A}$ and V B, we have tackled the problem of partitioning ecological signed networks with both competition and facilitation by simply analyzing the equilibrium distributions and compositions of the synchronization clusters. The identification of modules within the community and the position of species within those modules is central to understand the structure and functioning of ecological networks, independent of the type of biotic interactions considered. ${ }^{69}$

The application of synchronization to group partitioning in ecological networks also provides useful insights about the nodes and groups which play a major role in structuring the community. As shown in Sec. V C, this information helps us to explain the diversity and composition of vegetal patches and, more importantly, to anticipate the possible changes that the removal of species introduces in this organization. In this regard, the analysis of synchronization patterns allows to monitor how is the transition from the original organization to the emerging new one, thus providing a fast and useful framework to assess the resilience of ecological networks and to design accurate and focused conservation/restoration strategies.

\section{ACKNOWLEDGMENTS}

We are grateful to Concepción L. Alados and Yolanda Pueyo who kindly shared their data about Monegros (CGL2012-37508) and Cabo de Gata (CGL2011-27259). J.G.G. acknowledges financial support from the European Commission through FET IP projects MULTIPLEX (Grant No. 317532) and PLEXMATH (Grant No. 317614), the Spanish MINECO through the Ramón y Cajal program and Projects FIS2015-71582-C2 and FIS2014-55867-P, from the Departamento de Industria e Innovación del Gobierno de Aragón y Fondo Social Europeo (FENOL group E-19), and the Brazilian CNPq through the grant PVE of the Ciencias Sem Fronteiras program. HS acknowledges funding via a
JAEPre grant from the National Research Council of Spain. RFSA and FSB acknowledge financial support from FAPESB (Contract No. PNX 0006/2009) and by CNPQ (INCT-SC).

\section{APPENDIX: LINEAR STABILITY OF THE SYNCHRONIZED SOLUTIONS IN THE TWO POPULATIONS SYSTEM}

From the Kuramoto equations, Eq. (6), we can write the linearized equations governing the time evolution of small perturbations $\epsilon_{i}^{\alpha} \ll 1$ to the phases $\theta_{i}^{\alpha}$ as

$$
\begin{aligned}
\dot{\epsilon}_{i}^{\alpha}= & \sum_{j=1}^{N} K r^{\alpha} \cos \left(\Psi^{\alpha}-\theta_{i}^{\alpha}\right)\left[\frac{\partial \Psi_{i}^{\alpha}}{\partial \theta_{j}^{\alpha}}-\delta_{i j}\right] \epsilon_{j}^{\alpha} \\
& +\sum_{j=1}^{N} K \sin \left(\Psi^{\alpha}-\theta_{i}^{\alpha}\right) \frac{\partial r^{\alpha}}{\partial \theta_{j}^{\alpha}} \epsilon_{j}^{\alpha}+p K r^{\beta} \cos \left(\Psi^{\beta}-\theta_{i}^{\alpha}\right) \epsilon_{i}^{\alpha} \\
& -\sum_{j=1}^{N} p K r^{\beta} \cos \left(\Psi^{\beta}-\theta_{i}^{\alpha}\right) \frac{\partial \Psi_{i}^{\beta}}{\partial \theta_{j}^{\beta}} \epsilon_{j}^{\beta} \\
& -\sum_{j=1}^{N} p K \sin \left(\Psi^{\beta}-\theta_{i}^{\alpha}\right) \frac{\partial r^{\beta}}{\partial \theta_{j}^{\beta}} \epsilon_{j}^{\beta}
\end{aligned}
$$

where $\delta_{i j}$ is the Kronecker's delta function defined as $\delta_{i j}=1$ if $i=j$ and $\delta_{i j}=0$ otherwise.

Now, we consider an initial perturbation of the form $\epsilon_{i}^{\alpha}(0)=\delta$ and $\epsilon_{i}^{\beta}(0)=-\delta$ (with $\alpha=1,2$ and $\beta=2,1$, respectively), i.e., we perturb all the phases in one population in the same way while the opposite perturbation is applied to all the phases in the other one. This way, the perturbations in population $\alpha$ initially evolve as:

$$
\dot{\epsilon}_{i}^{\alpha}=2 p K r^{\beta} \cos \left(\Psi^{\beta}-\theta_{i}^{\alpha}\right) \delta,
$$

whereas those in population $\beta$ initially are as follows:

$$
\dot{\epsilon}_{i}^{\beta}=-2 p K r^{\alpha} \cos \left(\Psi^{\alpha}-\theta_{i}^{\beta}\right) \delta .
$$

Let us note that we have used these two equalities

$$
\begin{aligned}
& \sum_{j=1}^{N} \frac{\partial r^{\alpha}}{\partial \theta_{j}^{\alpha}}=0 \\
& \sum_{j=1}^{N} \frac{\partial \Psi^{\alpha}}{\partial \theta_{j}^{\alpha}}=1 .
\end{aligned}
$$

Now, we consider the first of the solutions to Eq. (8), $\Psi^{\alpha}=\Psi^{\beta}$ $=0\left(\theta_{i}^{\alpha}=\theta_{i}^{\beta}\right.$ for those oscillators with the same identical frequency $\omega_{i}^{\alpha}=\omega_{i}^{\beta}$ ), and set (by invoking the symmetry of the two populations system) that $r^{\alpha}=r^{\beta}$. Then we obtain from Eqs. (A2) and (A3): $\dot{\epsilon}_{i}^{\alpha}=-\dot{\epsilon}_{i}^{\beta}>0$, so that the phases of the oscillators $\theta_{i}^{\alpha}$ and $\theta_{i}^{\beta}$ separate from each other, thus pointing out that $\Psi^{\alpha}=\Psi^{\beta}=0$ is an unstable equilibrium.

Instead, substituting in Eqs. (A2) and (A3), the solution $\Psi^{\alpha}=0$ and $\Psi^{\beta}=\pi\left(\theta_{i}^{\alpha}=\theta_{i}^{\beta}-\pi\right.$ for those oscillators with the same identical frequency $\omega_{i}^{\alpha}=\omega_{i}^{\beta}$ ) yields: $\dot{\epsilon}_{i}^{\alpha}=-\dot{\epsilon}_{i} \beta<0$, so that the phases in both populations tend to recover their original position, i.e., they are stable. 
${ }^{1}$ A. Pikovsky, M. Rosenblum, and J. Kurths, Synchronization: A Universal Concept in Nonlinear Sciences (Cambridge University Press, 2003).

${ }^{2}$ S. C. Manrubia, A. S. Mikhailov, and D. H. Zanette, Emergence of Dynamical Order: Synchronization Phenomena in Complex Systems (World Scientific, 2004).

${ }^{3}$ S. Boccaletti, The Synchronized Dynamics of Complex Systems (Elsevier, 2008).

${ }^{4}$ D. J. Watts and S. H. Strogatz, "Collective dynamics of small-world networks," Nature 393, 440 (1998).

${ }^{5}$ S. H. Strogatz, "Exploring complex networks," Nature 410, 268 (2001).

${ }^{6} \mathrm{E}$. Bullmore and O. Sporns, "The economy of brain network organization," Nat. Rev. Neurosci. 10, 186 (2009).

${ }^{7}$ S. Boccaletti, V. Latora, Y. Moreno, M. Chavez, and D.-U. Hwang, "Complex networks: Structure and dynamics," Phys. Rep. 424, 175 (2006).

${ }^{8}$ A. Arenas, A. Díaz-Guilera, J. Kurths, Y. Moreno, and C. Zhou, "Synchronization in complex networks," Phys. Rep. 469, 93 (2008).

${ }^{9}$ T. Nishikawa, A. E. Motter, Y. C. Lai, and F. C. Hoppensteadt, "Heterogeneity in oscillator networks: Are smaller worlds easier to synchronize?," Phys. Rev. Lett. 91, 014101 (2003).

${ }^{10}$ Y. Moreno and A. F. Pacheco, "Synchronization of Kuramoto oscillators in scale-free networks," Europhys. Lett. 68, 603 (2004).

${ }^{11}$ M. Chavez, D.-U. Hwang, A. Amann, H. G. E. Hentschel, and S. Boccaletti, "Synchronization is enhanced in weighted complex networks," Phys. Rev. Lett. 94, 218701 (2005).

${ }^{12}$ A. Arenas, A. Díaz-Guilera, and C. J. Pérez-Vicente, "Synchronization reveals topological scales in complex networks," Phys. Rev. Lett. 96, 114102 (2006).

${ }^{13}$ C. Zhou, A. E. Motter, and J. Kurths, "Universality in the synchronization of weighted random networks," Phys. Rev. Lett. 96, 034101 (2006).

${ }^{14}$ J. Gómez-Gardeñes, Y. Moreno, and A. Arenas, "Paths to synchronization in complex networks," Phys. Rev. Lett. 98, 034101 (2007).

${ }^{15}$ I. Lodato, S. Boccaletti, and V. Latora, "Synchronization properties of network motifs," Europhys. Lett. 78, 28001 (2007).

${ }^{16}$ J. Gómez-Gardeñes, S. Gómez, A. Arenas, and Y. Moreno, "Explosive synchronization transitions in scale-free networks," Phys. Rev. Lett. 106, 128701 (2011).

${ }^{17}$ L. V. Gambuzza, A. Cardillo, A. Fiasconaro, L. Fortuna, J. GómezGardeñes, and M. Frasca, "Analysis of remote synchronization in complex networks," Chaos 23, 043103 (2013).

${ }^{18}$ T. Gross and B. Blasius, "Adaptive coevolutionary networks: A review," J. R. Soc. Interface 5, 259 (2008).

${ }^{19} \mathrm{C}$. Zhou and J. Kurths, "Dynamical weights and enhanced synchronization in adaptive complex networks," Phys. Rev. Lett. 96, 164102 (2006).

${ }^{20} \mathrm{~F}$. Sorrentino and E. Ott, "Adaptive synchronization of dynamics on evolving complex networks," Phys. Rev. Lett. 100, 114101 (2008).

${ }^{21}$ T. Aoki and T. Aoyagi, "Co-evolution of phases and connection strengths in a network of phase oscillators," Phys. Rev. Lett. 102, 034101 (2009).

${ }^{22}$ R. Gutierrez, A. Amann, S. Assenza, J. Gómez-Gardeñes, V. Latora, and S. Boccaletti, "Emerging meso-and macroscales from synchronization of adaptive networks," Phys. Rev. Lett. 107, 234103 (2011).

${ }^{23}$ A. Buscarino, L. Fortuna, M. Frasca, and A. Rizzo, "Dynamical network interactions in distributed control of robots," Chaos 16, 015116 (2006).

${ }^{24}$ M. Frasca, A. Buscarino, A. Rizzo, L. Fortuna, and S. Boccaletti, "Synchronization of moving chaotic agents," Phys. Rev. Lett. 100, 044102 (2008).

${ }^{25}$ N. Fujiwara, J. Kurths, and A. Díaz-Guilera, "Synchronization in networks of mobile oscillators,” Phys. Rev. E 83, 025101 (2011).

${ }^{26}$ L. Prignano, O. Sagarra, and A. Díaz-Guilera, "Tuning synchronization of integrate-and-fire oscillators through mobility," Phys. Rev. Lett. 110, 114101 (2013).

${ }^{27}$ J. Gómez-Gardeñes, V. Nicosia, R. Sinatra, and V. Latora, "Motioninduced synchronization in metapopulations of mobile agents," Phys. Rev. E 87, 032814 (2013).

${ }^{28} \mathrm{H}$. Daido, "Quasientrainment and slow relaxation in a population of oscillators with random and frustrated interactions," Phys. Rev. Lett. 68, 1073 (1992).

${ }^{29}$ J. C. Stiller and G. Radons, "Dynamics of nonlinear oscillators with random interactions," Phys. Rev. E 58, 1789 (1998).

${ }^{30} \mathrm{H}$. Daido, "Algebraic relaxation of an order parameter in randomly coupled limit-cycle oscillators," Phys. Rev. E 61, 2145 (2000).

${ }^{31}$ I. Leyva, I. Sendiña-Nadal, J. A. Almendral, and M. A. F. Sanjuán, "Sparse repulsive coupling enhances synchronization in complex networks," Phys. Rev. E 74, 056112 (2006).
${ }^{32}$ T. Nishikawa and A. E. Motter, "Network synchronization landscape reveals compensatory structures, quantization, and the positive effect of negative interactions," Proc. Nat. Acad. Sci USA 107, 10342 (2010).

${ }^{33}$ H. Hong and S. H. Strogatz, "Kuramoto model of coupled oscillators with positive and negative coupling parameters: An example of conformist and contrarian oscillators," Phys. Rev. Lett. 106, 054102 (2011).

${ }^{34}$ H. Hong and S. H. Strogatz, "Conformists and contrarians in a Kuramoto model with identical natural frequencies," Phys. Rev. E 84, 046202 (2011).

${ }^{35}$ H. Hong and S. H. Strogatz, "Mean-field behavior in coupled oscillators with attractive and repulsive interactions," Phys. Rev. E 85, 056210 (2012).

${ }^{36}$ D. Anderson, A. Tenzer, G. Barlev, M. Girvan, T. M. Antonsen, and E. Ott, "Multiscale dynamics in communities of phase oscillators," Chaos 22, 013102 (2012).

${ }^{37}$ R. M. Callaway and L. R. Walker, "Competition and facilitation: a synthetic approach to interactions in plant communities,” Ecology 78, 1958 (1997).

${ }^{38}$ M. R. Aguiar and O. E. Sala, "Patch structure, dynamics and implications for the functioning of arid ecosystems," Trends Ecol. Evol. 14, 273 (1999).

${ }^{39}$ F. S. Bacelar, J. M. Calabrese, and E. Hernández-García, "Exploring the tug of war between positive and negative interactions among savanna trees: Competition, dispersal, and protection from fire," Ecol. Complexity 17, 140 (2014).

${ }^{40} \mathrm{H}$. Saiz and C. L. Alados, "Changes in semi-arid plant species associations along a livestock grazing gradient," PloS One 7, e40551 (2012).

${ }^{41} \mathrm{R}$. Tirado and F. Pugnaire, "Community structure and positive interactions in constraining environments," Oikos 111, 437 (2005).

${ }^{42}$ Y. Kuramoto, "Self-entrainment of a population of coupled nonlinear oscillators," Lect. Notes in Physics 30, 420 (1975).

${ }^{43}$ S. H. Strogatz, "From Kuramoto to Crawford: Exploring the onset of synchronization in populations of coupled oscillators," Physica D 143, 1 (2000).

${ }^{44}$ J. A. Acebrón, L. L. Bonilla, C. J. Pérez-Vicente, F. Ritort, and R. Spigler, "The Kuramoto model: A simple paradigm for synchronization phenomena," Rev. Mod. Phys. 77, 137 (2005).

${ }^{45}$ J. G. Restrepo, E. Ott, and B. R. Hunt, "Onset of synchronization in large networks of coupled oscillators," Phys. Rev. E 71, 036151 (2005).

${ }^{46}$ F. Heider, "Attitudes and cognitive organization," J. Psychol. 21, 107 (1946).

${ }^{47}$ D. Cartwright and F. Harary, "Structure balance: A generalization of Heider's theory," Psych. Rev. 63, 277 (1956).

${ }^{48}$ M. Szell, R. Lambiotte, and S. Thurner, "Multirelational organization of large-scale social networks in an online world," Proc. Nat. Acad. Sci. USA 107, 13636 (2010)

${ }^{49}$ H. Saiz and C. L. Alados, "Effect of livestock grazing in the partitions of a semiarid plant-plant spatial signed network," Acta Oecol. 59, 18 (2014).

${ }^{50}$ H. Saiz, C. L. Alados, and Y. Pueyo, "Plant-plant spatial association networks in gypsophilous communities: the influence of aridity and grazing and the role of gypsophytes in its structure," Web Ecol. 14, 39 (2014).

${ }^{51}$ L. A. Cavieres, M. T. Arroyo, A. Peñaloza, M. A. Molina-Montenegro, and C. Torres, "Nurse effect of Bolax gummifera cushion plants in the alpine vegetation of the Chilean Patagonian Andes," J. Veg. Sci. 13, 547 (2002).

${ }^{52}$ L. A. Cavieres, E. I. Badano, A. Sierra-Almeida, S. Gómez-González, and M. A. Molina-Montenegro, "Positive interactions between alpine plant species and the nurse cushion plant Laretia acaulis do not increase with elevation in the Andes of central Chile," New Phytol. 169, 59 (2006).

${ }^{53}$ J. Gómez-Gardeñes, Y. Moreno, and A. Arenas, "Synchronizability determined by coupling strengths and topology on complex networks," Phys. Rev. E 75, 066106 (2007).

${ }^{54}$ S. Boccaletti, M. Ivanchenko, V. Latora, A. Pluchino, and A. Rapisarda, "Detecting complex network modularity by dynamical clustering," Phys. Rev. E 75, 045102(R) (2007).

${ }^{55}$ J. A. Almendral, I. Leyva, D. Li, I. Sendiña-Nadal, S. Havlin, and S. Boccaletti, "Dynamics of overlapping structures in modular networks," Phys. Rev. E 82, 016115 (2010).

${ }^{56}$ I. Sendiña-Nadal, Y. Ofran, J. A. Almendral, J. M. Buldú, I. Leyva, D. Li, S. Havlin, and S. Boccaletti, "Unveiling protein functions through the dynamics of the interaction network," PLoS ONE 6, e17679 (2011).

${ }^{57} \mathrm{E}$. Montbrió, J. Kurt's, and B. Blasius, "Synchronization of two interacting populations of oscillators,” Phys. Rev. E 70, 056125 (2004). 
${ }^{58}$ A. Rad, I. Sendiña-Nadal, D. Papo, M. Zanin, J. M. Buldú, F. del Pozo, and S. Boccaletti, "Topological measure locating the effective crossover between segregation and integration in a modular network," Phys. Rev. Lett. 108, 228701 (2012).

${ }^{59}$ D. Li, I. Leyva, J. A. Almendral, I. Sendiña-Nadal, J. M. Buldú, S. Havlin, and S. Boccaletti, "Synchronization interfaces and overlapping communities in complex networks," Phys. Rev. Lett. 101, 168701 (2008).

${ }^{60} \mathrm{~S}$. Boccaletti, G. Bianconi, R. Criado, C. I. Del Genio, J. GómezGardeñes, M. Romance, I. Sendiña-Nadal, Z. Wang, and M. Zanin, "The structure and dynamics of multilayer networks," Phys. Rep. 544, 1 (2014).

${ }^{61}$ M. Kivela, A. Arenas, M. Barthelemy, J. P. Gleeson, Y. Moreno, and M. A. Porter, "Multilayer networks," J. Complex Netw. 2, 203 (2014).

${ }^{62}$ See supplementary material at http://dx.doi.org/10.1063/1.4952960 for the linear stability analysis of the solutions to Eq. (8).

${ }^{63}$ R. W. Brooker, F. T. Maestre, R. M. Callaway, C. L. Lortie, L. A. Cavieres et al., "Facilitation in plant communities: the past, the present, and the future," J. Ecol. 96, 18 (2008).
${ }^{64}$ S. Soliveres, C. Smit, and F. T. Maestre, "Moving forward on facilitation research: response to changing environments and effects on the diversity, functioning and evolution of plant communities," Biol. Rev. 90, 297 (2015).

${ }^{65}$ A. B. Robles and C. B. Passera, "Native forage shrub species in southeastern Spain: forage species, forage phytomass, nutritive value and carrying capacity," J. Arid Environ. 30, 191 (1995).

${ }^{66}$ D. G. Milchunas and W. K. Lauenroth, "Quantitative effects of grazing on vegetation and soils over a global range of environments," Ecol. Monogr. 63, 327 (1993).

${ }^{67}$ C. L. Alados, P. Gotor, P. Ballester, D. Navas, J. M. Escos, T. Navarro, and B. Cabezudo, "Association between competition and facilitation processes and vegetation spatial patterns in alpha steppes," Biol. J. Linn. Soc. 87, 103 (2006).

${ }^{68}$ J. Wu, L. Zhang, Y. Li, and Y. Jiao, "Partition signed social networks via clustering dynamics," Physica A 443, 568 (2016).

${ }^{69}$ T. C. Ings, J. M. Montoya, and J. Bascompte et al., "Review: Ecological networks beyond food webs,” J. Animal Ecol. 78, 253 (2009). 